\title{
Task-relevant grasp selection: a joint solution to planning grasps and manipulative motion trajectories
}

\author{
Amir M. Ghalamzan E., Nikos Mavrakis, Marek Kopicki, Rustam Stolkin, Ales Leonardis
}

\begin{abstract}
This paper addresses the problem of jointly planning both grasps and subsequent manipulative actions. Previously, these two problems have typically been studied in isolation, however joint reasoning is essential to enable robots to complete real manipulative tasks. In this paper, the two problems are addressed jointly and a solution that takes both into consideration is proposed. To do so, a manipulation capability index is defined, which is a function of both the task execution waypoints and the object grasping contact points. We build on recent state-of-the-art grasp-learning methods, to show how this index can be combined with a likelihood function computed by a probabilistic model of grasp selection, enabling the planning of grasps which have a high likelihood of being stable, but which also maximise the robot's capability to deliver a desired post-grasp task trajectory. We also show how this paradigm can be extended, from a single arm and hand, to enable efficient grasping and manipulation with a bi-manual robot. We demonstrate the effectiveness of the approach using experiments on a simulated as well as a real robot.
\end{abstract}

\section{INTRODUCTION}

Grasping and manipulative motion planning have both been extensively studied in the robotics literature. However, because each of these problems is complex, the bulk of the literature has tackled them separately. Hence, a computed optimal grasp may not be efficient to perform a given manipulation task. For example, a state-of-the-art grasp planning algorithm may achieve a stable grasp on an object, but with a hand position which makes it impossible for the robot arm to deliver that object to its intended destination. In contrast, this paper addresses the problem of selecting a grasp that is likely to be stable, but which also results in the maximum capability of the robot to subsequently deliver a desired postgrasp manipulative trajectory. A comparatively small part of the previous literature jointly considers the problems of grasping and manipulation. In [1] the processes of grasp contact selection, grasp force optimization and manipulator arm/hand trajectory planning are formulated as a combined optimal control problem. However, the problem formulation in [1] requires an initial optimization for acquiring an object's grasp contact points, followed by a post-grasp optimization to find the optimal object path that can be followed, given the

A. Ghalamzan, N. Mavrakis, M. Kopicki, R. Stolkin are with the University of Birmingham. A A.Ghalamzanesfahani, nxm504, M.Kopicki, R.Stolkin, A.Leonardis\} @bham.ac.uk. This research was supported by: "EPSRC Feasibility Study: Machine-learning of Vision-guided Bi-manual Grasps, for Adaptable Autonomous Manipulation in Manufacturing Environments"; H2020 RoMaNS "Robotic Manipulation for Nuclear Sort and Segregation", 645582; EPSRC UK-Korea Civil Nuclear Collaboration "Robotic system for retrieving contaminated materials from hazardous zones", EP/M026477/1. We also acknowledge MoD/Dstl and EPSRC for providing the grant to support the UK academics (Ales Leonardis) involvement in a Department of Defense funded MURI project.

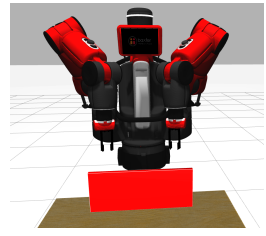

(a)

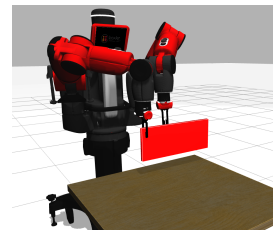

(c)

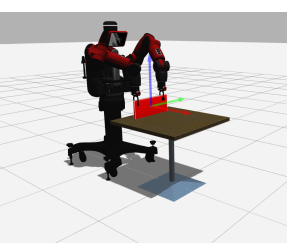

(b)

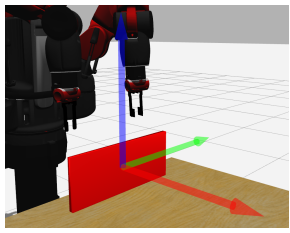

(d)
Fig. 1. (a) Screen-shot of the Baxter robot in Gazebo, a simulator with physics engine. The Baxter robot at its neutral configuration; (b) The robot grasps and manipulates a cuboid object. The $x, y, z$ world coordinate frame axes correspond with the red, green and blue axes shown; (c) The Baxter robot at the pick configuration of the Pick-and-Place task; (d) Closer view of the object showing the local frame $x, y, z$.

optimal grasping configuration. Our approach operates in an opposite manner, which is finding the optimal grasp points that maximise the manipulation capability for the subsequent desired task trajectory.

Furthermore, [1] also adopts a classical approach to grasping, based on fingertip point contacts and force-closure analysis, which assumes knowledge of the grasped object's geometry, mass, friction coefficients and other information a-priori, typically unknown in real grasping problems. The method is demonstrated using a simplified simulation of a 2 dof arm plus 2-finger hand, constrained to move in a plane. Even in this simplified example, the algorithm (complexity $O\left(n^{4}\right)$ and $O\left(m^{3}\right)$ where $n$ and $m$ are the number of finger and arm joints) takes fifteen minutes to converge on a solution. In contrast, our work is built on a recent state-ofthe-art learning-based grasp planner [2], which can generate a space of possible grasps on arbitrarily shaped objects, where all parts of the palm and fingers may contact the grasped object in complex ways. Our proposed method for choosing task-relevant grasps is computationally cheap, and we demonstrate it working in 3D examples of bi-manual grasping using the Baxter robot's two arms of 7dofs each.

In [3], a multi-phase manipulation task is learned from human demonstrations. A multi-phase model state-based transitions autoregressive hidden Markov model (STARHMM) was used to learn a distribution over sub-phases of a demonstrated task. The "phases" comprise actions such as 
approaching the object with the left and right arm of a humanoid robot, grasping, lifting and moving the object. A set of motor primitive policies are then learned for each phase of the task, and reinforcement learning is used to make the transition between phases. This approach thus considers grasping and subsequent manipulative motion as two separate stages, and the example task included execution of grasping and manipulation, but with no dependency or optimization between the two actions. In contrast, our paper shows how to choose the grasp positions specifically so as to improve the robot's ability to perform the subsequent manipulative actions.

For the problem of grasping, a variety of grasp planning approaches have been proposed. One category of methods [4], [5] includes those which search for shapes on the object that fit within the robot gripper. Another category of methods includes those that allow the robot to learn a mapping between visual input and action [6]. The latter case has been examined in [2], where a probabilistic approach was proposed for providing a robot with a space of likely grasps.

The approach of [2] models the hand configuration with respect to the shape of the object using a single demonstrated grasp example. The learned model is a product of two kernel density functions: a density over the pose of a single hand link relative to the local object surface, and a density over the whole hand configuration. This approach captures the local relation between hand and object shape, resulting in an optimal grasp. However, as far as manipulation is concerned, the computed grasp may lack performance in terms of manipulation capability.

For the problem of measuring manipulation capability, measuring indices such as manipulability and reachability have been specifically considered in the context of dualarm manipulation, with motivation stemming from the need to manipulate bulky and heavy objects. Zacharias et al. [7] presented an efficient way of assessing the reachability of different parts of the workspace, and used this to help choose an easiest-to-reach grasp position. Vahrenkamp et al. [8] showed how a robot holding an object in one hand, could efficiently move its other hand to achieve a bi-manual grasp. In contrast to our work, both of these methods relied on pre-programmed grasps already being known a-priori for a specific object. Neither of the methods explained how to choose grasps to facilitate a specific desired post-grasp manipulative motion. [9] and [10] extended this work by introducing new measures of manipulability, and showing how they could be used to choose bi-manual grasps resulting in highly manipulable configurations. However, these later works also relied on a database of pre-known grasps for each object, and also did not address the main problem tackled in this paper, namely how to plan grasps which are chosen so as to facilitate a specific desired post-grasp task motion. In contrast, [9], [10] proposed an optimal grasp resulting in a maximum manipulability at initial grasp configuration.

The main contributions of this paper are as follows:

- we propose new measures of manipulation capability which are specific to a particular desired task trajectory;
- we show how this approach can be extended from uni-manual to bi-manual or multi-arm grasping and manipulation problems;

- we demonstrate the usefulness of the proposed approach, using both uni-manual and bi-manual examples of pick-and-place and pick-rotate-place tasks with the Baxter robot.

\section{LEARNING AND GENERATION OF GRASPS FOR ARBITRARILY SHAPED OBJECTS}

[2] showed how grasp models can be trained using only a single demonstrated grasp on a single object, but enable new grasps to be computed for new objects of arbitrary shape. This grasp learning and generation approach relies on probabilistic modelling of surface features, extracted from $3 \mathrm{D}$ depth images. The surface features $x \in S E(3) \times \mathbb{R}^{2}$ consist of curvature $r \in \mathbb{R}^{2}$ and a local frame attached to surface point ${ }^{g c} \mathbf{x} \in S E(3)$, where $S E(3)$ denotes the group of $3 \mathrm{D}$ poses (3D position $p$ and $3 \mathrm{D}$ orientation $q$ ). $S E(3)=\mathbb{R}^{3} \times$ $S O(3)$ and $S O(3) \subset \mathbb{R}^{3 \times 3}$ denotes the group of rotations in three dimensions:

$$
S O(n)=\left\{R \in \mathbb{R}^{n \times n}: R R^{T}=I, \operatorname{det}(R)=+1\right\} .
$$

The object model is defined as a joint probability distribution of a set of features, modelled as kernel density estimation:

$$
\left.O\left({ }^{g c} \mathbf{x}, r\right)=\operatorname{pdf}^{o}{ }^{g c} \mathbf{x}, r\right) \simeq \sum_{j=1}^{K_{0}} w_{j} \mathcal{K}\left({ }^{g c} \mathbf{x}, r \mid x_{j}, \sigma_{x}\right)
$$

where $\mathrm{w}_{j} \in \mathbb{R}^{+}$are kernel weights, and

$$
\mathcal{K}\left({ }^{g c} \mathbf{x}, r \mid x_{j}, \sigma_{x}\right)=\mathcal{N}_{3}\left(p \mid \mu_{p}, \sigma_{p}\right) \theta\left(q \mid \mu_{q}, \sigma_{q}\right) \mathcal{N}_{2}\left(r \mid \mu_{r}, \sigma_{r}\right)
$$

$\mu$ and $\sigma$ are the kernel mean and kernel bandwidth. $\mathcal{N}_{*}$ is an $*$-variate isotropic Gaussian kernel, and $\theta$ is a Gaussian-like distribution in $S O(3)$.

Contact model $\operatorname{pdf}_{i}^{M}(u, r)$ encodes the joint probability distribution of surface features and of the $3 \mathrm{D}$ pose of the $i_{t h}$ hand link, where $u_{i j}=v_{j}^{-1} \circ s_{i}, s_{i}$ and $\circ$ denote the pose of $L_{i}$ and the composition operator. The contact model of link $L_{i}$ is

$$
\mathcal{B}_{i}(u, r)=\frac{1}{Z} \sum_{j=1}^{K_{0}} \mathcal{N}_{3}\left(p \mid \mu_{p}, \sigma_{p}\right) \theta\left(q \mid \mu_{q}, \sigma_{q}\right) \mathcal{N}_{2}\left(r \mid \mu_{r}, \sigma_{r}\right)
$$

where $Z \in \mathbb{R}^{+}, u_{i j}=\left(p_{i j}, q_{i j}\right)$ is the pose of link $L_{i}$ relative to the pose of $v_{j}$ of the $j^{\text {th }}$ surface feature.

Hand configuration model $C$ weights the space of hand configurations to favour configurations most similar to those observed during training. $C$ is constructed as a kernel density as follows:

$$
\mathcal{C}\left(h_{c}\right)=\sum w\left(h_{c}(\gamma)\right) \mathcal{N}\left(h_{c} \mid h_{c}(\gamma), \sigma_{c}\right)
$$

where, $w\left(h_{c}(\gamma)\right)=\exp \left(-\alpha\left\|h_{c}(\gamma)-h_{c}^{g}\right\|^{2}\right), \alpha$ is a real positive value, $h_{c}(\gamma)=(1-\gamma) h_{c}^{g}+\gamma h_{c}^{t}$ and $\gamma \in[-\beta, \beta], \beta \in \mathbb{R}^{+}$. $h_{c}^{g}$ and $h_{c}^{t}$ denote joint angles at some small distance before the hand reached the training object, and the joint angles at the time the hand makes a contact with the training object. Query density $Q_{i}$ is a density over possible $i^{\text {th }}$ link poses $s$ 
given a new object point cloud. $Q_{i}$ is computed by convolving the corresponding contact model $B_{i}$ with a new object point cloud $O$.

$$
Q_{i}(s) \simeq \sum_{j=1}^{K_{Q_{i}}} w_{i j} \mathcal{N}_{3}\left(p \mid \hat{p}_{i j}, \sigma_{p}\right) \theta\left(q \mid \hat{q}_{i j}, \sigma_{q}\right)
$$

To generate a grasp for a new object, a finger link is selected at random, and a link pose is sampled from the query density. A hand configuration $h_{c}$ is then sampled from $C$. Hence, the corresponding hand pose is determined using the hand configuration $h_{c} \in \mathbb{R}^{D}$ and arm configuration $h_{w} \in \mathbb{R}^{D_{r}}$ $\left({ }^{g c} \mathbf{x}=F K\left(h_{w}\right)\right)$. We now compute the likelihood of a grasp using the kernels

$$
\mathcal{L}(h)=\mathcal{L}_{C}(h) \mathcal{L}_{Q}(h)=C\left(h_{c}\right) \prod_{Q_{i} \in Q} Q_{i}\left(F K_{i}\left(h_{w}, h_{c}\right)\right)
$$

where $h=\left(h_{w}, h_{c}\right)$ and $\mathrm{FK}_{i}$ denotes the forward kinematics corresponding to $i_{\text {th }}$ link of the hand. The objective of grasp optimization is to find a grasp that maximizes the product of the likelihood of the query densities and the hand configuration density

$$
\bar{h}=\operatorname{argmax} \mathcal{L}(h)
$$

Given an example of a successful grasp, the kernels can be computed. Consequently, a set of grasp candidates can be sampled from the kernels and the corresponding likelihoods can be computed using eq. (3). Hence, this model provides us with a space of possible grasp solutions and the corresponding likelihoods. A set of likely grasp candidates will be used in the next sections for further analysis resulting in grasps optimal both in terms of contacts of robot's hand fingers and object surface as well as in terms of robot manipulation capability.

\section{SELECTING GRASPS THAT MAXIMISE TASK-RELEVANT MANIPULABILITY}

In this section, we explain how to search the solution space of likely grasps (eq. (3)) to select the grasp that is most useful for a subsequent manipulation task. In order for a robot to manipulate an object, the robot's end-effector visits a sequence of poses mapping to a corresponding sequence of poses which we desire the manipulated object to visit. The sequence of object poses are determined by task constraints. For example, during a task of manipulating a teapot to pour tea into a tea cup, a robot's end effector movements are constrained by the desired tea pot pose needed to achieve successful pouring. A number of different approaches have been proposed for planning the sequence of poses that the robot's end-effector must visit to complete a task, such as RRT planing [8], [11], [12] and learning from demonstration [13]. Throughout this paper, we assume that a sequence of optimal robot end-effector poses can be found, either by using Dynamic Movement Primitives [13] or an appropriate planning algorithm.

We denote by $\left\{{ }_{c} O,{ }_{c} x,{ }_{c} y,{ }_{c} z\right\}$ a local frame ${ }^{c} \mathbf{x}$ attached to the center of mass $c$ of the object to be manipulated (Fig. 2). Let us consider a task of picking an object at an initial

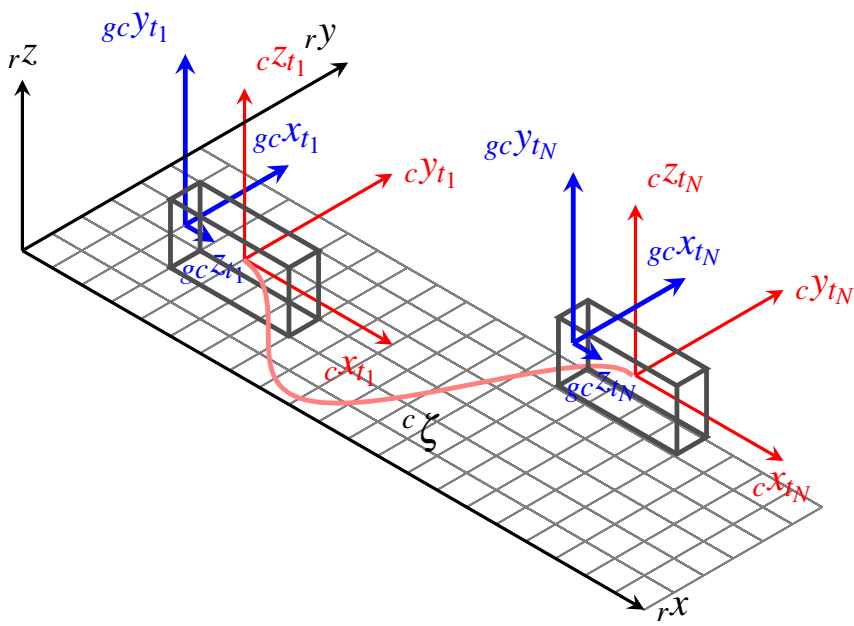

Fig. 2. An object in the global coordinate frame ${ }^{r} \mathbf{x}=\left\{{ }_{r} O,{ }_{r} x,{ }_{r} y,{ }_{r} z\right\}$, shown in black. A local coordinate frame ${ }^{c} \mathbf{x}=\left\{{ }_{c} O,{ }_{c} x_{c} y,{ }_{c} z\right\}$ is attached to the center of mass of the object, shown in red color. This frame follows a trajectory ${ }^{c} \boldsymbol{\zeta}$ during manipulation. ${ }^{c} \mathbf{x}_{t_{1}}=\left\{{ }_{c} O_{t_{1}}, c_{t_{1}},{ }_{c} y_{t_{1}},{ }_{c} z_{t_{1}}\right\}$ and ${ }^{c} \mathbf{x}_{t_{n}}=$ $\left\{{ }_{c} O_{t_{n}},{ }_{c} x_{t_{n}}, c y_{t_{n}}, c z_{t_{n}}\right\}$ denote this frame at the initial and terminal point of the manipulation trajectory with the corresponding frame of grasp candidate ${ }^{g c} \mathbf{x}_{t_{1}}=\left\{{ }_{g c} O_{t_{1}},{ }_{g c} x_{t_{1}},{ }_{g c} y_{t_{1}},{ }_{g c} z_{t_{1}}\right\}$ and ${ }^{g c} \mathbf{x}_{t_{n}}=\left\{{ }_{g c} O_{t_{n}},{ }_{g c} x_{t_{n}},{ }_{g c} y_{t_{n}},{ }_{g c} z_{t_{n}}\right\}$ shown with blue color.

pose and placing it at a target pose, see Fig. 1 We consider four steps for this task (Fig. 11):

- locating the robot's end-effector at the pre-grasp pose and then grasping the object;

- picking the object;

- moving the object to the target pose;

- placing the object at the target pose.

These four steps correspond with four waypoints ${ }^{c} \zeta=$ $\left\{{ }^{c} \mathbf{x}_{\text {initial }},{ }^{c} \mathbf{x}_{\text {pick }},{ }^{c} \mathbf{x}_{\text {move }},{ }^{c} \mathbf{x}_{\text {place }}\right\}$.

\section{A. Problem formulation}

Let us denote a world reference frame $\left\{{ }_{r} O,{ }_{r} x, r_{r} y,{ }_{r} z\right\}$ by ${ }^{r} \mathbf{x}$. A trajectory to be followed by the manipulated object implies that local frame ${ }^{c} \mathbf{x}$ follows a sequence of poses:

$$
\begin{gathered}
{ }^{c} \boldsymbol{\zeta}={ }^{c} \mathbf{x}(t) \\
0 \leq t \leq \mathrm{T}
\end{gathered}
$$

where $t$ denotes the time and $\mathrm{T}$ is the total time that the robot needs to complete the manipulation task $1{ }^{c} \zeta$ determines a complete object pose at every time $t$. Although there are different possible representations of orientation, for the sake of simplicity here we use the conventional transformation matrix.

Let us consider again local frame ${ }^{c} \mathbf{x}=\left\{{ }_{c} O,{ }_{c} x,{ }_{c} y,{ }_{c} z\right.$. This frame can be determined by a transformation matrix ${ }^{2}$ from the global reference frame $\left\{{ }_{r} O,{ }_{r} x,{ }_{r} y,{ }_{r} z\right\}$ into the local frame

\footnotetext{
${ }^{1}$ Throughout this paper, $Y(t)$ denotes a continuous function of time, where $Y_{i}$ is a corresponding value of $Y(t)$ at time $t_{i} \forall i=1, \ldots, n$, where $t_{1}=0, t_{n}=\mathrm{T}$ and $0 \leq t_{i} \leq \mathrm{T}$ is discrete sampling time. We also use $Y_{t}$ as a shorthand of $Y(t)$ if necessary. ${ }^{*} \mathbf{x}(t)$ and ${ }^{*} \mathbf{x}_{t}$ are continuous and discrete trajectory of poses of a frame attached to point $*$ of object in Figs. 2 3(a) and 3

${ }^{2}$ In general, ${ }_{(.)}^{(.)} \mathbf{x} \in \mathbb{R}^{4 \times 4}$ denotes a transformation matrix from local frame (.) into local frame (..).
} 


$$
\begin{aligned}
& \left\{{ }_{c} O,{ }_{c} x,{ }_{c} y,{ }_{c} z\right\}: \\
& \begin{array}{r}
{ }^{c} \mathbf{x}(t)={ }_{r}^{c} T(t) \\
{ }_{r}^{c} T(t)=\left[\begin{array}{r:c}
R_{3 \times 3}(t) & d_{3 \times 1}(t) \\
\hdashline 0_{1 \times 3} & 1
\end{array}\right] .
\end{array}
\end{aligned}
$$

Given a sampling time, $\left\{t_{1}, \ldots, t_{n}\right\}$, a discrete-time trajectory, corresponding with Eq. (5) is represented by a sequence of homogenous transformations:

$$
{ }^{c} \zeta=\left\{{ }_{r}^{c} T_{1},{ }_{r}^{c} T_{2}, \ldots,{ }_{r}^{c} T_{n}\right\}
$$

where, ${ }_{r}^{c} T_{i}$ is the transformation matrix at time $t_{i}$ with corresponding rotation $R_{i}=R\left(t_{i}\right)$ and translation $d_{i}=d\left(t_{i}\right)$. The corresponding local frames are ${ }^{c} \mathbf{x}_{i}={ }^{c} \mathbf{x}\left(t_{i}\right) \forall i=1, \ldots, n$, where, $t_{1}=0, t_{n}=\mathrm{T}$. Note that here we assume grasping and manipulation of rigid objects. Extending our proposed methods to deformable objects will make an interesting problem for future work.

Let us denote a local frame attached to the robot wrist by ${ }^{g c} \mathbf{x}=\left\{{ }_{g c} O,{ }_{g c} x,{ }_{g c} y,{ }_{g c} z\right\}$ which corresponds with the arm configuration $h_{w}$. Because the object is non-deformable, any robot wrist pose candidate can be expressed by a fixed transformation matrix ${ }_{c}^{g c} T$ from ${ }^{c} \mathbf{x}$ into ${ }^{g c} \mathbf{x}$ (Fig. 3):

$$
\begin{array}{r}
{ }^{g c} \mathbf{x}_{i}={ }_{c}^{g c} T \times{ }^{c} \mathbf{x}_{i}, \\
\forall{ }^{g c} \mathbf{x}_{i} \in{ }^{g c} \mathcal{X}, \\
i_{g c}=1, \ldots, n_{g c}
\end{array}
$$

where ${ }^{g c} \mathcal{X}$ is a set of wrist poses for all likely grasps, corresponding with the set of arm configuration $h_{w}$ computed by eq. 3 . Using ${ }^{c} \zeta$ in eq. (5), the trajectory of poses followed by ${ }^{c} \mathbf{x}$, and eq. (8), we can now write the trajectory of ${ }^{g c} \mathbf{x}$ as follows:

$$
\begin{array}{r}
{ }^{g c} \zeta=\left\{{ }_{c}^{i_{g c}} T \times{ }^{c} \mathbf{x}_{1},{ }_{c}^{i_{g c}} T \times{ }^{c} \mathbf{x}_{2}, \ldots,{ }_{c}^{i_{g c}} T \times{ }^{c} \mathbf{x}_{n}\right\} \\
g c \zeta={ }_{c}^{i_{g c}} T \times{ }^{c} \zeta,
\end{array}
$$

We consider that ${ }^{g c} \zeta \in{ }^{g c} \mathcal{Z}$, where ${ }^{g c} \mathcal{Z}$ denotes a group of pose trajectories corresponding with the task of manipulating the object $\mathcal{O}$ in Fig. 2 and $\forall^{g c} \mathbf{x} \in{ }^{g c} \mathcal{X}$ in Fig. 3(a). Given a grasp candidate ${ }^{g c} \mathbf{x}$ and ${ }^{c} \mathbf{x}$, one can easily compute ${ }_{c}^{g c} T$. Therefore, ${ }^{g c} \mathcal{Z}$ can be compactly represented by

$$
{ }^{g c} \mathcal{Z}={ }_{c}^{g c} \mathcal{T} \times{ }^{c} \zeta
$$

where, ${ }_{c}^{g c} \mathcal{T}$ is a group of transformation matrices from ${ }^{c} \mathbf{x}$ into ${ }^{g c} \mathcal{X}$.

\section{B. Task relevant kinematic manipulability}

Let us consider an object $\mathcal{O}$ in a robot workspace, a set of valid grasp candidates ${ }^{g c} \mathcal{X}$ and the corresponding likelihoods provided by the grasp learning and generation approach of [2]. By valid grasp candidate we mean a robotic hand pose relative to the object to be grasped whose corresponding arm configuration exists. In the context of robotics, manipulability is known as a measure of robot's capability to perform manipulations, defined in [14] as follows:

$$
m(J)=\sqrt{\operatorname{det}\left(J J^{T}\right)}=\left(\lambda_{1} \lambda_{2} \ldots \lambda_{n_{D}}\right)^{\frac{1}{2}}
$$
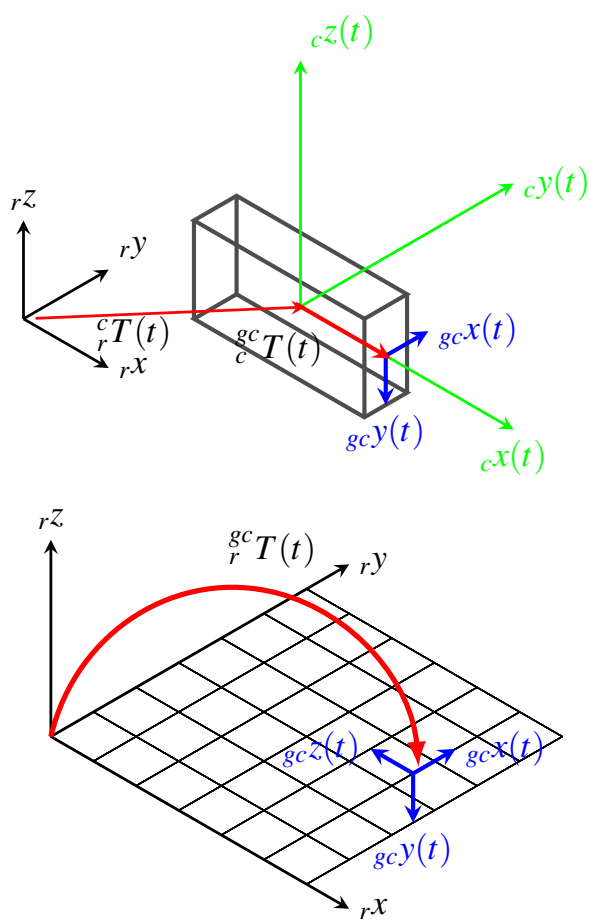

Fig. 3. Top: a non-deformable object is shown in the global frame. At time $t,{ }^{c} \mathbf{x}(t)=\left\{{ }_{c} o(t),{ }_{c} x(t),{ }_{c} y(t),{ }_{c} z(t)\right\}$ is attached to the centre of mass of the object to be manipulated. Frame ${ }^{c} \mathbf{x}(t)$ is expressed by a transformation matrix ${ }_{c}^{r} T$ from ${ }^{r} \mathbf{x}$ to ${ }^{c} \mathbf{x}$. At every time, a frame attached to the object ${ }^{g c} \mathbf{x}(t)=\left\{{ }_{g} c o(t),{ }_{g_{c}} x(t),{ }_{g c} y(t),{ }_{g_{c}} z(t)\right\}$ can always be expressed by a single homogeneous transformation ${ }_{c}^{{ }^{c} c} T$ from ${ }^{c} \mathbf{x}(t)$ into ${ }^{g c} \mathbf{x}(t)$. Note that ${ }_{c}^{g c} T$ is not a function of time $t$; Bottom: a total transformation from ${ }^{g c} \mathbf{x}=\left\{{ }_{g c} \mathrm{O},{ }_{g c} x,{ }_{g c} y,{ }_{g c} z\right\}$ into ${ }^{r} \mathbf{x}=\left\{{ }_{r} o,{ }_{r} x,{ }_{r} y,{ }_{r} z\right\}$.

where, $\lambda_{j}$ is $j_{t h}$ eigenvalue of $J J^{T}$, and $n_{D}$ denotes the dimension of output-space. The manipulability in eq. (11) is proportional to the volume of manipulability ellipsoid.

For the sake of coherence, let us consider the relation of the Jacobian and the notion of manipulability to be used in this paper. The basic kinematics of a robot is expressed by $\dot{x}=J(q) \dot{q}$. Using virtual work, one can also derive the relation between force at the end-effector and torques of the joint as follows:

$$
\tau=J^{T} F
$$

where $\tau, J$, and $F$ are joint torques, Jacobian and generalised force applied at end-effector, respectively. The applied torques which generate a desired generalised force at the endeffector can be computed using the following equation:

$$
F=\left(J J^{T}\right)^{-1} J \tau
$$

where $J J^{T}$ is the basis of manipulability definitions. This matrix and its inverse are positive definite if they do not have a zero eigenvalue. A positive definite matrix maps a unit circle input to an ellipse in the output space. The eigenvalues and eigenvectors of $J J^{T}$ form a manipulability ellipsoid (Fig. 4). The capability of manipulation index along the eigenvector of $J J^{T}$ corresponding to the minimum eigenvalue is less than the corresponding value along the other eigenvectors. Hence, inverse of condition number was introduced in [15], as follows:

$$
c(J)=\frac{1}{\operatorname{cond}(J)}=\frac{\lambda_{n_{D}}}{\lambda_{1}}
$$




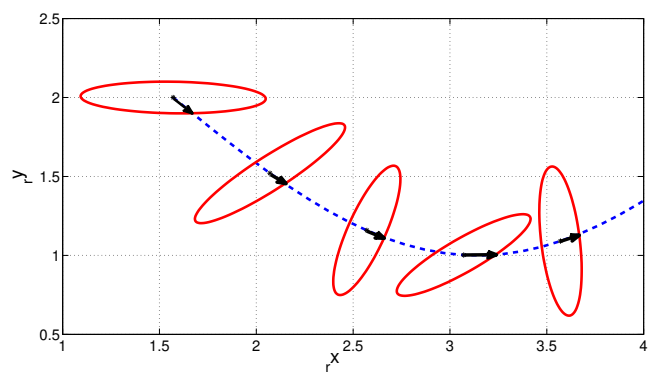

Fig. 4. A schematic of manipulability at different poses of a robot's end effector. Blue dashed line represents a path of the end-effector poses. At each pose of the end-effector, the manipulability ellipsoid consists of the principal axes of $J J^{T}$ multiplied by the corresponding eigenvalues. The manipulability ellipsoid is shown with red line and task relevant manipulability is shown with black arrows.

A value of $c(J)$ close to one implies an isotropic ellipsoid. This means that manipulator has equivalent capability of manipulation along all directions. More discussion about force and velocity manipulability as well as Dynamically Weighted Kinematic Manipulability and the extension to Eq. (12) can be found in [16]. Moreover, a joint limit and an obstacle penalty function were discussed to modify the manipulability ellipsoid in [10].

In many manipulation tasks, a desired trajectory of an object is known a priori. Given a trajectory of the object to be manipulated ${ }^{c} \boldsymbol{\zeta}$ and given the wrist poses of a single grasp that has been executed on the object, we can compute a trajectory of the end-effector ${ }^{3}$ poses ${ }^{g c} \boldsymbol{\zeta}$ using eq. (10) and the corresponding arm configurations using inverse kinematics of the arm. A trajectory of Jacobians $J(t){ }^{4}$ is then computed corresponding with ${ }^{g c} \boldsymbol{\zeta}$. We are interested in manipulability that expresses the manipulation capability of the robotic arm along the corresponding object trajectory. Therefore, we define manipulability along the movement (Fig. 4) as follows:

$$
M_{T}\left(J(t),{ }^{g c} \boldsymbol{\zeta}\right)=\sum_{j=1}^{n_{D}} \lambda_{j}\left(\mathrm{v}_{j} \cdot U_{g c} \boldsymbol{\zeta}\right)^{2}
$$

where $\mathrm{v}_{j}$ is $j_{t h}$ eigenvector of $J J^{T}, M_{T}\left(t,{ }^{g c} \boldsymbol{\zeta}\right) \in \mathbb{R}$ and $U_{g c} \boldsymbol{\zeta}$ is a unit vector tangent to trajectory of robot end-effector pose ${ }^{g c} \boldsymbol{\zeta}$ at time $t$. In eq. (13), $M_{T}\left(J(t),{ }^{g c} \boldsymbol{\zeta}\right)$ represents the projection of manipulability ellipsoid on a line tangent to the corresponding trajectory. We consider this to be representative of robot capability along that direction.

Different norms of this signal can now be computed as the task relevant manipulability based on task requirements, for instance one can integrate the manipulability index over the execution time:

$$
\boldsymbol{M}\left({ }_{c}^{g c} T\right)=\int_{0}^{T} M_{T}(t) d t
$$

\footnotetext{
${ }^{3}$ We use the terms end-effector and wrist interchangeably throughout this paper since the trajectory of end-effector can be computed based on the trajectory of the wrist and the corresponding transformation matrix.

${ }^{4}$ Here we assume that either we can compute the Jacobian analytically or that the same Jacobian used by the real robot is available for grasp computation.
}

\section{Task relevant grasp selection}

We first formalize the task relevant grasp selection for the case of single arm, and then we describe how to extend this formulation to multi-arm scenarios.

1) Single arm Manipulation:: Let us consider again the sampling time $t_{1}, \ldots, t_{n} . M_{T}(t)$ in eq. (13) becomes:

$$
\mu=\left[M_{T}\left(t_{1},{ }_{c}^{g c} T\right), M_{T}\left(t_{2},{ }_{c}^{g c} T\right), \ldots, M_{T}\left(t_{n_{D}},{ }_{c}^{g c} T\right)\right],
$$

and $U_{g c} \boldsymbol{\zeta}_{\left(t_{i}\right)}$ is a unit vector computed by a homogeneous transformation matrix that transforms ${ }^{g c} \mathbf{x}_{i}$ into ${ }^{g c} \mathbf{x}_{i+1}{ }^{5}$. Given a vector of manipulability along the corresponding trajectory in eq. [15], we can compute different norms of $\mu$, as follows:

$$
\begin{array}{r}
\left.\mu_{l_{\infty}}{ }^{g c} \mathbf{x}\right)=\left\|\left[\frac{1}{M_{T}\left(t_{1},{ }_{c}^{g c} T\right)}, \ldots, \frac{1}{M_{T}\left(t_{n_{D}},{ }^{g c} T\right)}\right]\right\|_{\infty} \\
\mu_{l_{2}}\left({ }^{g c} \mathbf{x}\right)=\left\|\left[M_{T}\left(t_{1},{ }_{c}{ }_{c}^{g c} T\right), \ldots, M_{T}\left(t_{n_{D}},{ }_{c}^{g c} T\right)\right]\right\|_{2}
\end{array}
$$

We may also approximate the integral in eq. (14) using trapezoidal approximation:

$$
\mu_{l_{1}}\left({ }^{g c} \mathbf{x}\right)=\frac{1}{2} \sum_{i=1}^{n-1}\left(\left(M_{T}\left(t_{i}\right)+M_{T}\left(t_{i+1}\right)\right) \Delta t_{i}\right)
$$

We are interested to select a grasp candidate which not only maximizes the grasp likelihood based on demonstration and local object shape, but also results in a higher task-relevant manipulability.

2) Dual/multi-arm Manipulation: Here we extend the formulation to a scenario of dual/multi-arm grasping and manipulation. In the case of multi-arm grasping, a set of valid grasp candidates ${ }^{g c} \mathcal{X}_{k_{r}} \subset G P \forall k_{r}=1, \ldots, K_{r}$ where $K_{r}$ is the number of robotic arms, must exist. We extend the model to the multi-arm problem. Given an object in the robot workspace, a grasp candidate can be expressed as in eq. (8).

$$
{ }^{g c} \mathbf{x}_{k_{r}}={ }_{c}^{g c} T_{k_{r}} \times{ }^{c} \mathbf{x} \forall k_{r}=1, \ldots, K_{r} .
$$

Using eq. 9), the corresponding trajectories can be written as:

$$
{ }^{g c} \zeta_{k_{r}}={ }_{c}^{g c} T_{k_{r}} \times{ }^{c} \zeta \forall k_{r}=1, \ldots, K_{r}
$$

Then, the corresponding manipulability along the trajectory, $\mu_{*, k_{r}}(h) \forall k_{r}=1, \ldots, K_{r}$, is computed using eq. (15). The computed manipulability is then used to find the optimal grasp poses by eq. 16.

\section{EXPERIMENTAL RESULTS}

We set up an experimental configuration using a Baxter robot ${ }^{\circledR}$ to demonstrate the effectiveness of the proposed approach. The Baxter robot, manufactured by Rethink Robotics, includes a torso based on a movable pedestal and two 7-DOF arms. Each arm has 7 rotational joints and 8 links. The robot comes with an electric parallel jaw gripper and a vacuum cup which can be installed at the end of each arm. For our experiment, we used Baxter SDK along with PyKDL and Gazebo simulation 6 For the demonstration of the method,

\footnotetext{
${ }^{5}$ For the sake of simplicity we do not use different notations to express different pose representations. For example, ${ }^{g c} \mathbf{x}_{i}$ stands for all pose representations including different orientation definitions, such as quaternion, rotation matrix and Euler angles.

${ }^{6}$ More information can be found at http://sdk.rethinkrobotics.com/wiki.
} 


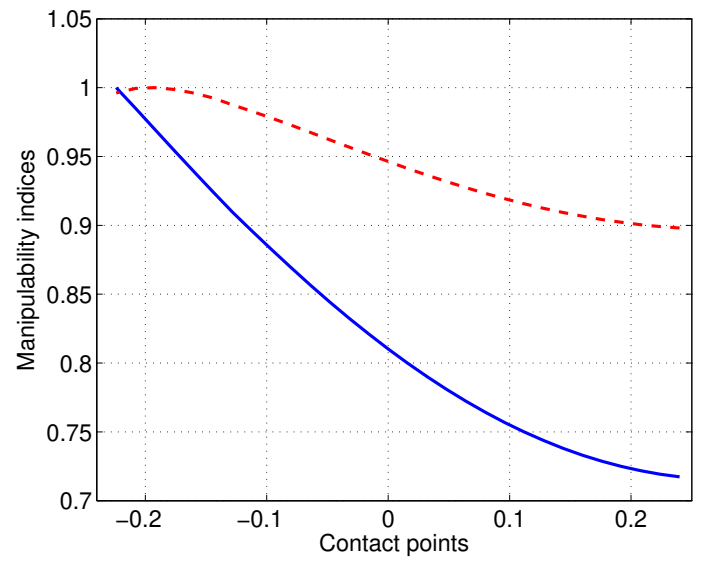

Fig. 5. Two manipulability indices of the left arm normalized over their corresponding maximum value which are proposed in this work, namely $\mu_{l_{\infty}}$ and $\mu_{l_{2}}$ are shown with blue line and red dashed line, respectively. Based on these two indices, the best grasping contact points are located at the left-most part of the object. The decline of the two lines demonstrates that grasping contact points on the object further on the right side reduces the manipulability indices over the path. $\mu_{l_{2}}$ is used as the main index to be maximized where $\mu_{l_{\infty}}$ is used as a constraint, i.e. we avoid selecting grasping contact points that yield large values for second index $\mu_{l_{\infty}}>\mu_{0}$.

we conducted two sets of simulation and real experiment. In simulation experiments the Baxter robot manipulates a cuboid-object whereas in real experiment it manipulates a T-shape object, as shown in Fig. 11 and Fig. 10, respectively.

The previous work [2] showed how to plan and execute grasps using arbitrarily complex hands, on objects with complex and completely arbitrary shapes, without any apriori knowledge of the objects' geometry, using only a single view from a depth camera. In this paper, we use the Baxter to perform manipulation tasks of cuboid object in Gazebo simulation environment and manipulation tasks of a T-shaped object with a cylindrical handle. These experiments with the simple Baxter gripper demonstrate proof of principle of the main contribution of the paper - explaining how to choose grasps, which maximise subsequent task-relevant manipulability.

\section{A. Simulation experiments}

The simulation experiments include two tasks of manipulating a cuboid-object: Pick-and-Place and Pick-RotatePlace. In order to reduce the computational complexity, we only consider the set of grasp candidates along the top edge of the cuboid example object, however our method can be applied to grasps anywhere on an arbitrarily shaped object in general. All these top contacts have equivalent grasp likelihood, which is higher than the grasp likelihoods for contacts anywhere else on the object. From the robot's perspective, the positive y-axis corresponds with the left side and the negative with the right. The first contact point is located on the left-most region and the last contact point on the right-most region.

In the Pick-and-Place experiment, with respect to the robot coordinate frame, the Baxter robot picked up the object along the z-axis, moved it to the left, along the y axis, and placed

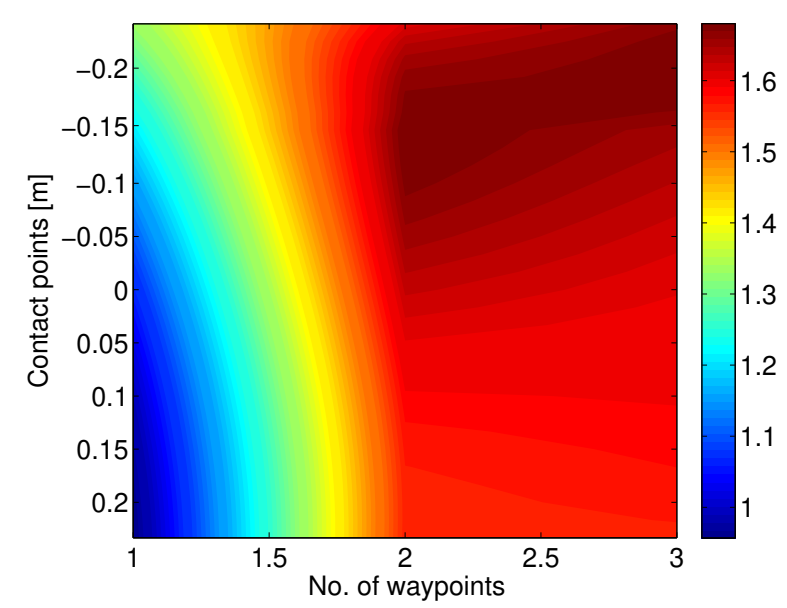

Fig. 6. $\mu_{l_{2}}$ of the left arm normalized over its maximum value versus all contact points on the object and the waypoints of the Pick-and-Place task. This index increases for the first waypoint from the far right of the object (positive value of the contact coordinates), to the far left of the object (negative value of the contact coordinates). Red regions correspond to high manipulability index.

it on the table along the negative z-axis (Fig. 1). A set of grasp candidates can be generated using eq. 3 .

For the sake of simplicity, most figures show plots corresponding to the outcome of our proposed method using the left arm. The results for the Pick-and-Place experiment are shown in Fig. 5 where two different indices are provided, namely $L_{2}$ and $L_{\infty}$ norm of the task-relevant manipulability. The observed drop in both indices is justified by the fact that as soon as the robot uses the left arm and chooses a grasping point lying on the right side of the object, it reduces the manipulation capability of the movement along the left side. As a consequence, for the pick-and-place task the maximum manipulability comes from grasping the object from the left side. A more detailed visualization of the result is shown in Fig. 6, where the colourmap represents the manipulability $L_{2}$ index versus the task waypoints. In this figure, the highest index corresponds with the reddest colour, while blue colours denote lower index values. The manipulability is observed to reach higher values when the starting grasp point is located on the left side of the object. The first waypoint corresponds to the object lifting action so it is expected that the manipulability will be higher close to the neutral configuration of the robot (Fig. 1(a)). The second waypoint denotes the object's translation motion along the positive y-axis, resulting in the highest manipulability region of the map. This is expected because, when starting from the left side of the object and moving to the left, the robot endeffector pose is relatively close to the neutral configuration.

In Fig. 7. a general interpretation of the experimental result is given for both robot arms. This figure provides the sum of the manipulabilities for both left and right arm, when they start from the respective contact points. Based on this figure, the highest manipulability of dual arm manipulation implies that the left arm is on the left side of the object and the right arm is on the right side of the object. 


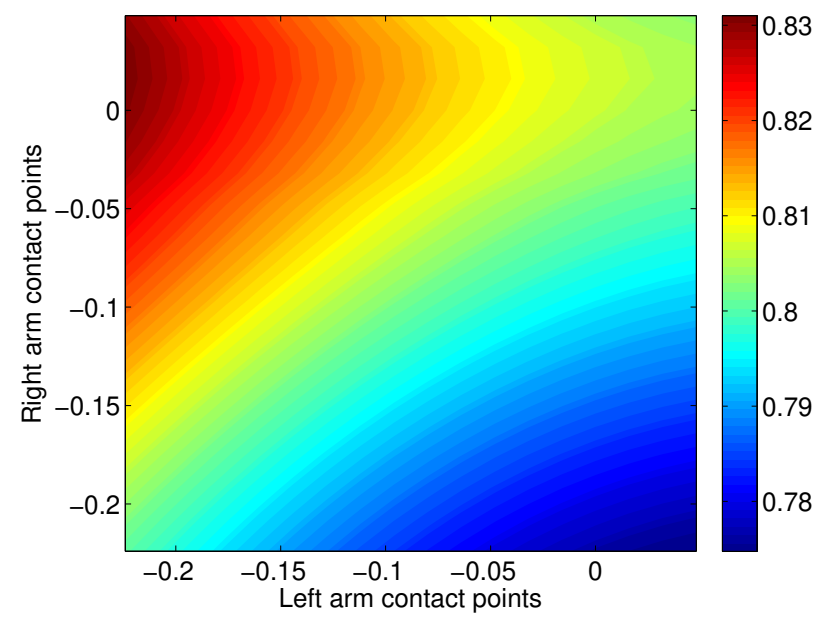

Fig. 7. The sum of $\mu_{l_{2}}$ for an example bi-manual manipulation. This index is normalized over its maximum value and it is depicted versus all contact points with the left and right arm in the Pick-and-Place task. The red region, towards the top left part of this figure, corresponds to a high manipulability of the sum indices implying that contact points of the robot's right hand at a mid-right location, and the robot's left hand at the left-most part of the object, result in the best manipulation capability. For extreme right regions of the object, no valid inverse kinematics solution were available and so they are omitted.

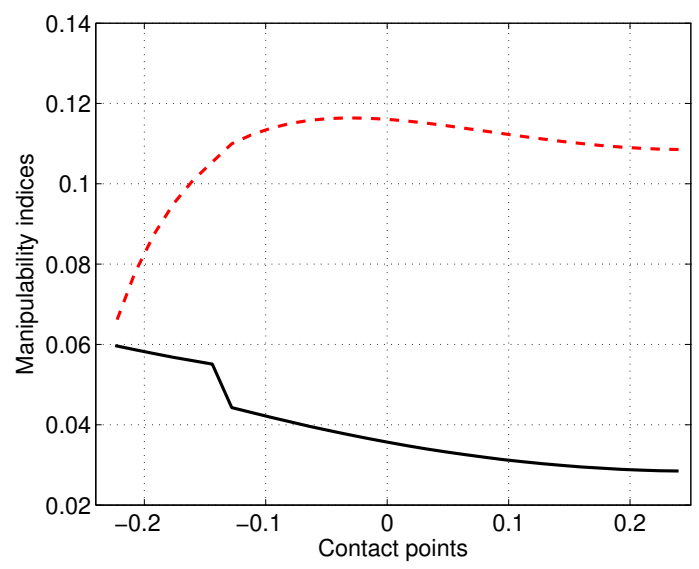

Fig. 8. Manipulability indices of the Pick-and-Place task with the left arm defined in eq. 11 and 12 correspond to the red dashed and black line, respectively. Both indices do not take into consideration the task waypoints for the calculation of the manipulability, resulting in a large difference between the computed maximum manipulability contact points.

To demonstrate the effectiveness of the method, we also compare against manipulability indices proposed in other literature [9], shown in Fig. 8. If these indices were to be used in the experiment, the robot would choose a different contact point for grasping. These contact points, however, are not optimal for a manipulative motion trajectory. They are computed only based on the arm configuration corresponding with a grasping pose.

To further examine and compare the performance of our proposed method for more complex arm configurations, we set up a secondary task, which consists of grasping the object, lifting it alongside the $\mathrm{z}$-axis, rotating it +90 degrees

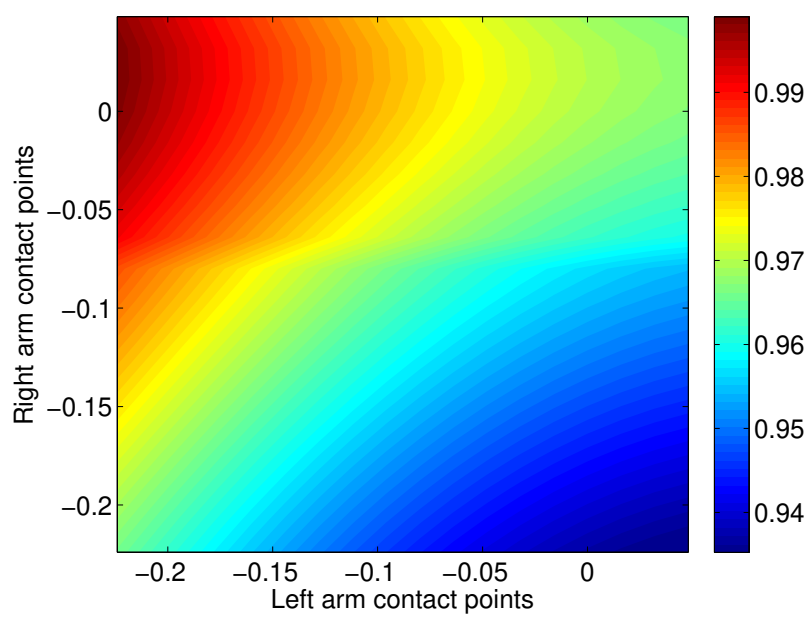

Fig. 9. The sum of $\mu_{l_{2}}$ for the two-arm manipulation in the Pick-RotatePlace task. This index is normalized over its maximum value and it is depicted versus all contact points of the left and right arms. The region with hot colours in the top-left part of the figure, corresponds with a high manipulation capability. Moving along the negative and positive value of $\mathrm{y}$-axis (along the top of the object) corresponds with gradient descent for left and right arms, respectively.

around the z-axis, and then putting it down again. The results are presented in Fig. 9 The behaviour of our method is in accordance with the previous experiment, i.e. the optimal configuration for hand placement includes the left hand on the left side of the object and the right hand on the right side of the object, as in Fig. 1. even though the task includes rotating the object. This outcome can be deduced from Fig. 9 which shows the colour map of the hand configuration on the object and the corresponding sum of the manipulability indices.

\section{B. Real Experiment}

We also set up some experiments with a real Baxter to exemplify the effectiveness of the proposed approach. In these experiments, the Baxter robot manipulated the object shown in Fig. 10(b) This object was specifically manufactured for this experiment. Three examples are presented here: Pick-Rotate-Place (Fig. 10(c) and 10(d)], Pick-MovePlace (Fig. 10(e) and 10(f)], and Pick-Move/Rotate-Place (Fig. $10(\mathrm{~g})$ and 10(h) . In all these experiments, the Baxter robot picked the object at initial pose shown in Fig. 10(a), manipulated it and placed it at the corresponding target poses. For all the experiments, a set of 16 grasp candidates were considered. Baxter's left arm at initial grasp configuration is shown in Fig. 10(b) The next grasp candidates were generated by rotating the end-effector $10^{\circ}$ counterclockwise around the z-axis normal to the top of the table. Fig. 10(d), 10(f) and 10(h) show the target poses of the object corresponding with a Pick-Rotate-Place, Pick-MovePlace and Pick-Move/Rotate-Place, respectively. Besides, Fig. 10(c), 10(e) and 10(g) show the best grasp poses and arm configurations resulting in the highest manipulability for performing the Pick-Rotate-Place, Pick-Move-Place and Pick-Move/Rotate-Place movements, respectively. Different 


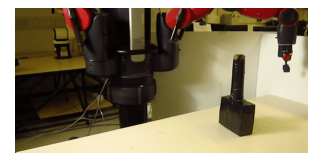

(a)

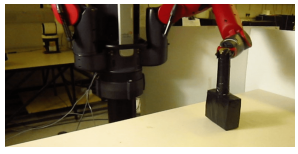

(c)

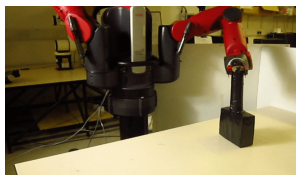

(e)

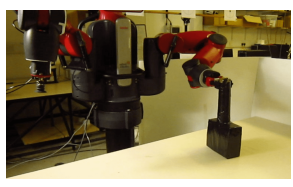

(g)

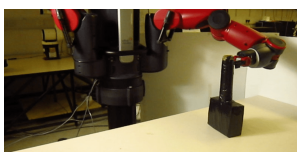

(b)

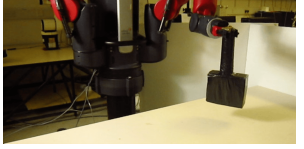

(d)

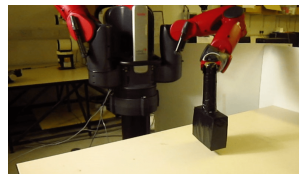

(f)

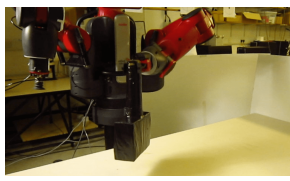

(h)
Fig. 10. The Baxter robot grasps the object at initial position ( shown in Fig. (b) and performs: (c) and (d) Pick-Rotate-Place, (e) and (f) Pick-MovePlace and Pick-Move-Rotate-Place (g) and (h) Figures in the left column show the best initial robot's arm configurations for the corresponding manipulation tasks according to the manipulability index introduced in eq. 16. Images on the right show the target pose of corresponding manipulation task. Fig. (a) shows the first grasp candidate in the predetermined set of grasps equally likely in terms of contact points.

grasp poses and initial configurations of the robot reveal that the manipulative motion effectively is considered for grasp selection. This may become very critical; for example, the Baxter may not be able to perform the task shown in Fig. $10(\mathrm{~g})$ and $10(\mathrm{~h})$ if it grasps the object at the pose shown in Fig. 10(c)

From these experiments it is evident that, for a given task, the manipulability index we defined is dependent not only on the starting grasp pose and the corresponding location on the object, but also on the nature of the task itself, making it a very effective method for choosing grasp candidates for an object in order to carry out a desired manipulation task.

\section{CONCLUSION}

This paper has proposed a combined solution to the problem of both computing grasp contact points and also maximizing the resulting manipulation capability. In much of the previous literature, the extraction of grasping contact points has been computed independently of the the desired post-grasp manipulation task. This results in sub-optimal solutions wherein the computed grasp contact points may result in poor manipulation capability, or may even result in singular arm configurations along the desired task path.

To overcome such problems, we first defined a new manipulation capability index, which depends on the grasping contact points and a desired task path for the object being manipulated. We then used the new manipulation capability index, by showing how it can be applied to a particular object and manipulation task, to find the grasping contact points which jointly maximize the grasping likelihood and the manipulation capability.

We demonstrated the effectiveness of the proposed approach by conducting two different simulation experiments using a Baxter robot. We also compared the performance achieved with our proposed manipulability index, against results achieved using a well known manipulability index from the literature. The experiments and comparisons show that the conventional approach results in the same grasping contact points on the object regardless of the desired task path, while, in contrast, our proposed manipulability index adapts the grasping contact points to the desired task manipulation trajectory.

Our future work will explore ways of extending these methods to cope with deformable materials and objects with uneven mass distributions.

\section{REFERENCES}

[1] M. B. Horowitz and J. W. Burdick, "Combined grasp and manipulation planning as a trajectory optimization problem," in IEEE International Conference on Robotics and Automation, 2012, pp. 584-591.

[2] M. Kopicki, R. Detry, M. Adjigble, R. Stolkin, A. Leonardis, and J. Wyatt, "One-shot learning and generation of dexterous grasps for novel objects," International Journal of Robotics Research, vol. 35, no. 8, pp. 959-976, 2016.

[3] O. Kroemer, C. Daniel, G. Neumann, H. Van Hoof, and J. Peters, "Towards learning hierarchical skills for multi-phase manipulation tasks," in IEEE International Conference on Robotics and Automation, 2015, pp. 1503-1510.

[4] M. Trobina and A. Leonardis, "Grasping arbitrarily shaped 3-d objects from a pile," in IEEE International Conference on Robotics and Automation, vol. 1, 1995, pp. 241-246.

[5] A. Ten Pas and R. Platt, "Localizing handle-like grasp affordances in 3d point clouds," in Experimental Robotics. Springer, 2016, pp. 623-638.

[6] R. Detry, C. H. Ek, M. Madry, and D. Kragic, "Learning a dictionary of prototypical grasp-predicting parts from grasping experience," in IEEE International Conference on Robotics and Automation, 2013 pp. 601-608.

[7] F. Zacharias, C. Borst, and G. Hirzinger, "Capturing robot workspace structure: representing robot capabilities," in IEEE/RSJ International Conference on Intelligent Robots and Systems, 2007, pp. 3229-3236.

[8] N. Vahrenkamp, D. Berenson, T. Asfour, J. Kuffner, and R. Dillmann, "Humanoid motion planning for dual-arm manipulation and regrasping tasks," in IEEE/RSJ International Conference on Intelligent Robots and Systems, 2009, pp. 2464-2470.

[9] N. Vahrenkamp, M. Przybylski, T. Asfour, and R. Dillmann, "Bimanual grasp planning," in IEEE-RAS International Conference on Humanoid Robots, 2011, pp. 493-499.

[10] N. Vahrenkamp, T. Asfour, G. Metta, G. Sandini, and R. Dillmann, "Manipulability analysis," in 12th IEEE-RAS International Conference on Humanoid Robots, 2012, pp. 568-573.

[11] N. Vahrenkamp, T. Asfour, and R. Dillmann, "Simo: A simulation and motion planning toolbox for c+," 2010.

[12] R. Diankov and J. Kuffner, "Openrave: A planning architecture for autonomous robotics," Robotics Institute, Pittsburgh, PA, Tech. Rep. CMU-RI-TR-08-34, vol. 79, 2008.

[13] S. Schaal, J. Peters, J. Nakanishi, and A. Ijspeert, "Learning movement primitives," in Robotics Research. The Eleventh International Symposium. Springer, 2005, pp. 561-572.

[14] T. Yoshikawa, "Manipulability of robotic mechanisms," The international journal of Robotics Research, vol. 4, no. 2, 1985, pp. 3-9.

[15] M. Togai, "An application of the singular value decomposition to manipulability and sensitivity of industrial robots," SIAM Journal on Algebraic Discrete Methods, vol. 7, no. 2, 1986, pp. 315-320.

[16] F. Park and J. W. Kim, "Manipulability of closed kinematic chains," Journal of mechanical design, vol. 120, no. 4, 1998, pp. 542-548. 